\title{
Confocal laser endomicroscopy in breast surgery
}

\author{
Giovanni D. De Palma ${ }^{1}$ - Gaetano Luglio ${ }^{1} \cdot$ Dario Esposito $^{1}$
}

Received: 4 October 2015/Accepted: 22 October 2015/Published online: 24 October 2015

(C) Springer Science+Business Media New York 2015

\section{Dear Editor,}

We have read with interest the paper entitled "Imaging breast cancer morphology using probe-based confocal laser endomicroscopy: towards a real-time intraoperative imaging tool for cavity scanning", by Dr Chang and coll., published in September 2015 in "Breast Cancer Research and Treatment" [1].

We agree with the authors that the role of the p-CLE as a diagnostic tool in breast cancer "has yet to be systematically evaluated ... and warrants further attention".

We strongly believe in the diagnostic potential of confocal laser endomicroscopy in surgical oncology and we would also like to note that our research group has actually already published preliminary data on the role of p-CLE in breast imaging [2]. In our original article entitled "Confocal laser endomicroscopy in breast surgery: a pilot study", published back in April 2015, we similarly describe morphological patterns at p-CLE of both breast cancer and normal tissue; moreover, we have also performed the confocal imaging on patients affected by benign breast diseases (e.g. fibroadenoma). Instead of using acriflavine to stain the sections, we used intravenous fluorescein as contrast agent, administered soon before surgical resection; we have also tried to find the best interval between fluorescein infusion and surgical resection to obtain good quality images. We found that a longer interval (with fluorescein infusion at least 5 min before surgical resection) provides a better fluorescein impregnation with consequent better quality images. This was particularly true in benign lesions, probably due to the hypovascularization of such lesions; we also argued how the relation between the fluorescein infusion interval and image quality might also be considered as a further criteria to discriminate between benign and malignant lesions [2].

These results, together with those achieved by Dr Chang and coll., emphasize the need for further research to definitively establish the role of pCLE in the field of breast cancer and assess its clinical implications.

Kind regards,

Prof. Giovanni Domenico De Palma, Dr. Gaetano Luglio, Dr. Dario Esposito.

\section{References}

1. Chang TP, Leff DR, Shousha S, Hadjiminas DJ, Ramakrishnan R, Hughes MR, Yang GZ, Darzi A (2015) Imaging breast cancer morphology using probe-based confocal laser endomicroscopy: towards a real-time intraoperative imaging tool for cavity scanning. Breast Cancer Res Treat 153(2):299-310

2. De Palma GD, Esposito D, Luglio G, Limite G, Accurso A, Sollazzo V, Maione F, Cassese G, Siciliano S, Gennarelli N, Ilardi G, Paternoster M, Giglio MC, Forestieri P (2015) Confocal laser endomicroscopy in breast surgery: a pilot study. BMC Cancer $15: 252$

Giovanni D. De Palma

giovanni.depalma@unina.it

1 School of Medicine, University of Naples Federico II, Naples, Italy 